\title{
Proposed shunt rounding technique for large- scale security constrained loss minimization
}

\author{
P.J. Macfie, G.A. Taylor, MIEEE, M.R. Irving, SMIEEE, P. Hurlock, H.-B. Wan
}

\begin{abstract}
Optimal reactive power flow applications often model large numbers of discrete shunt devices as continuous variables, which are rounded to their nearest discrete value at the final iteration. This can degrade optimality. This paper presents novel methods based on probabilistic and adaptive threshold approaches that can extend existing security constrained optimal reactive power flow methods to effectively solve large-scale network problems involving discrete shunt devices. Loss reduction solutions from the proposed techniques were compared to solutions from the mixed integer non-linear mathematical programming algorithm (MINLP) using modified IEEE standard networks up to 118 buses. The proposed techniques were also applied to practical large-scale network models of Great Britain. The results show that the proposed techniques can achieve improved loss minimization solutions when compared to the standard rounding method.
\end{abstract}

Index Terms - discrete shunt control, security constrained optimal reactive power flow, large-scale transmission networks, optimization methods, integer programming, optimal control, loss minimization, power system modelling, reactive power control

\section{INTRODUCTION}

$\mathrm{N}$ ational Grid is the system operator (SO) for the high voltage electricity transmission system in Great Britain (GB). The authors have demonstrated [1] that a theoretical $2.3 \%$ reduction in operational transmission losses was possible on the GB transmission system. These studies utilised a transmission loss reduction objective and were based on a security constrained optimal reactive power flow (SCORPF) which stipulated available operational voltage controls. The authors noted that large numbers of discrete shunt devices were being treated as continuous variables in the main optimization and were rounded to their nearest discrete step at the final iteration. This rounding method was found to degrade optimality and potentially create

This work was financially supported in part by the National Grid and the EPSRC.

P.J. Macfie is with National Grid, Wokingham and Brunel University, Uxbridge, UK (email: peter.macfie@uk.ngrid.com)

P. Hurlock is with National Grid, Wokingham, UK (email: paul.hurlock@uk.ngrid.com)

G.A. Taylor is with Brunel University, Uxbridge, UK (email: gareth.taylor@brunel.ac.uk)

M.R. Irving is with Brunel University, Uxbridge, UK (email: malcolm.irving@brunel.ac.uk)

H.-B. Wan is with Brunel University, Uxbridge, UK (email: hai.wan@brunel.ac.uk) infeasibilities. The practical GB transmission network includes around 270 shunt capacitors and reactors, which provide an important source of reactive power that does not need to be procured from the market as the equipment is owned and operated by National Grid. It is therefore important that shunt capacitor and reactor controls are included in the optimization problem. This paper proposes and compares several novel methods that can easily be incorporated into existing SCORPF programs to solve the discrete switching problem more effectively. First, a probabilistic method is described which fixes a subset of shunts to their nearest discrete value on each iteration with a probability determined by a shunts proximity to a discrete value. Second, an adaptive threshold method is described which sets shunts to their nearest discrete value if they are within a threshold. The proposed techniques are evaluated and compared using a range of standard test networks, including the Ward Hale six bus test systems in figure 1, and full large-scale models of the GB transmissions system with security constraints.. Recent literature [2, 6, 7, 8] argues that the discrete shunt switching problem essentially remains unsolved in a reasonable time on a complex largescale power system. This paper directly addresses this problem by describing novel alternative techniques, which can be readily implemented as an extension to existing ORPF programs, for solving the discrete shunt switching problem. The proposed technique is also shown to be securable against a number of contingencies.

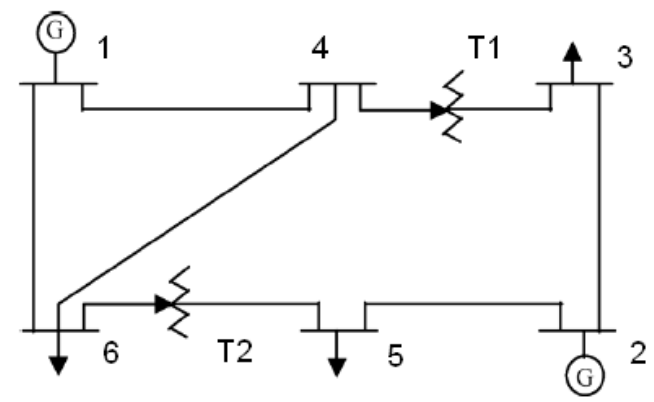

Fig. 1 Ward Hale six bus network test case.

\section{TECHNICAL BACKGROUND}

Most commercially available SCORPF programs still use methods based on rounding. Recent studies [2, 3] which were based on sections of the European grid used rounding methods and one of these studies focused on the Spanish transmission network. This study utilized rounding when the 
discrete step size was small, but needed to use an expert system heuristic to decide the appropriate position of large shunt capacitor/reactor devices.

An issue highlighted by previous studies is the problem of scalability of existing techniques to solve the large-scale shunt despatch problem $[14,4,15]$. The reason for this is likely to be due to the non-deterministic polynomial (NP) complete nature of the discrete capacitor despatch problem. A problem that is NP-complete is an adequate reason not to devote time and effort trying to solve it according to many experts [4]. Instead it is recommended that a near optimum solution to the problem is found. Many of the practical solutions in the literature only attempt to locate a near optimum solution. Non-deterministic search techniques include simulated annealing [17] and genetic algorithms [18]. The success of these algorithms is often highly dependant on tuning particular algorithmic parameters.

Deterministic search techniques include modern mixed integer solvers such as MINLP, which often use branchbound techniques to solve the generalized mixed integer problem. Jabr [4] suggested a technique that uses a modified version of the branch-bound technique to solve this mixed integer problem. The modification consisted of a heuristic that essentially limits the search space that the branch-bound algorithm needed to explore, which reduced the execution time of the mixed integer linear program solver. This technique was demonstrated to work in a reasonable time with only 25 integer variables, which is not scalable enough to solve problems on the practical large-scale GB transmission system.

To solve the mixed integer problem involving large numbers of shunt capacitors/reactors Liu et al. [5] proposed utilizing a penalty function associated with each discrete control. These penalty functions could be defined in such a way as to penalise continuous values so that the optimization would favour discrete solutions. Liu proposed taking a tangent to the quadratic at the current operating point. The linear tangent function needed to be updated periodically to preserve the quadratic nature of the penalty function. The main issues with this approach were in determining the magnitude of the penalty function, timing its introduction, determining criteria for updating, incorporating limit enforcement logic and determining the best technique for finally fixing to a discrete step. One further issue could be that the optimization may get trapped by the penalty function in a local minimum and would not therefore explore another potentially more optimal area of the solution space. A robust scalable solution is still sought after to solve the practical shunt despatch problem.

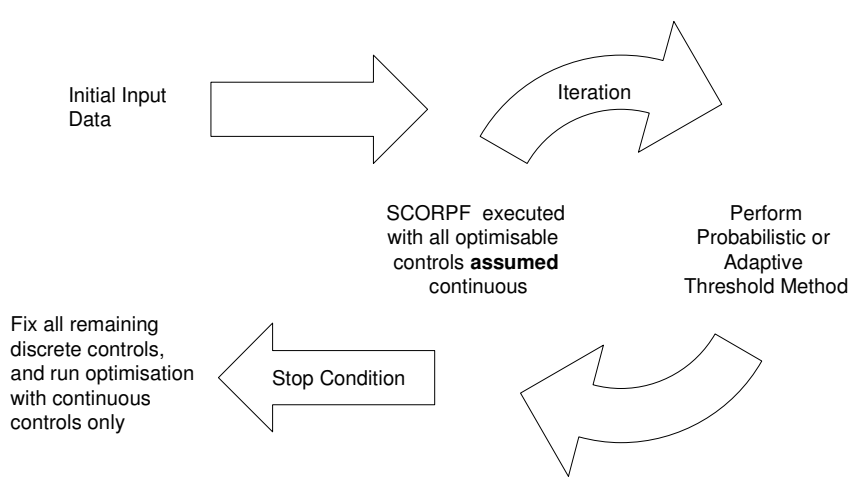

Fig. 2 Procedural chart of the enhanced SC-ORPF method - at each iteration a subset of shunts are fixed to their nearest discrete value as described in the text.

\section{PROPOSED METHOD}

\section{A. General Method for handling discrete variables}

Figure 2 illustrates how the iterative process proceeds. Firstly the network data is read and the main SCORPF is performed assuming all controls are continuous. At each iteration the optimal continuous solution from SCORPF is used to fix a subset of the floating shunt controls to their nearest discrete value, based on either the probabilistic or adaptive threshold techniques explained in the following sections. This process is then repeated iteratively until a stop condition is satisfied when either the maximum number of iterations is reached or when all discrete controls are fixed. Any remaining floating discrete controls are then fixed to their nearest discrete value. Finally a round of optimization is performed using only continuous controls to remove any violations caused by the final discretization.

In stage 1 the generalised continuous SCORPF objective function is:

$f\left(u^{0}, x^{0}\right)$

$u$ is a set of the control variables and $x$ is a set of dependent variables. The subscript 0 indicates that the variable refers to the pre-contingency power system.

Specifically this objective includes the total system losses given by:

$\operatorname{Losses}_{\text {Tot }}^{A C}=\sum_{l=1}^{N_{l}}-g_{i j}\left(V_{i}^{2}+V_{j}^{2}-2 V_{i} V_{j} \operatorname{Cos} \theta_{i j}\right)$

$l$ is a branch with conductance $g$ connecting the ith node to the $j$ th node, and $\mathrm{N}_{l}$ is the total number of branches on the network. $V$ and $\theta$ are node voltage magnitude and angle, respectively.

Initially, the SCORPF is bound by equality and inequality constraint limits. The equality constraint limits are given by the pre and post contingency power flow equations, where $k$ refers to the $k$ th contingency case. In general these equality constraints are given by: 
$g^{k}\left(u^{k}, x^{k}\right)=0 \quad$ for $k=0,1 \ldots n$

For the intact network case these power flow equations are given by the following equations [19]:

$$
\begin{aligned}
& P_{i j}=V_{i}^{2} g_{i j}-V_{i} V_{j}\left(g_{i j} \cos \left(\theta_{i}-\theta_{j}\right)+b_{i j} \sin \left(\theta_{i}-\theta_{j}\right)\right) \\
& Q_{i j}=-V_{i}^{2}\left(b_{i j}+b_{i j}^{s h}\right)-V_{i} V_{j}\left(g_{i j} \sin \left(\theta_{i}-\theta_{j}\right)-b_{i j} \cos \left(\theta_{i}-\theta_{j}\right)\right)
\end{aligned}
$$

$P_{i j}$ and $Q_{i j}$ are the real and reactive branch power flows respectively between the $i$ th and jth node. $g, b$ and $\theta$ are the conductance, susceptance and angle respectively between the $i$ th and $j$ th node. $b^{\text {sh }}{ }_{\text {ij }}$ is the branch's shunt susceptance.

The optimization is also constrained by inequality constraints. For optimisable controls these constraints are given by:

$$
u_{M I N}^{k} \leq u^{k} \leq u_{M A X}^{k}
$$

For dependant variables, such as voltage, these constraints are given by:

$$
x_{M I N}^{k} \leq x^{k} \leq x_{M A X}^{k}
$$

\section{B. Probabilistic Technique}

The probabilistic technique examines the continuous solution of each optimisable shunt control at every iteration of figure 2, fixing a subset of the shunts to the nearest discrete value. The probability of fixing is determined by how far the continuous solution is from the nearest discrete value on a normal distribution, as illustrated in Figure 3. Essentially if the continuous value of the capacitor is close to a discrete value there is a high chance of fixing and if a shunt is in the middle of the range of discrete values there is a low chance of fixing.

The probability distribution shown in Figure 3 can be used to calculate the probability that a shunt is fixed in and is based on the normal distribution which is given by:

$$
\begin{aligned}
\text { Scaled normal distribution } & =\frac{s}{\sigma \sqrt{2 \pi}} e^{-\frac{1}{2}\left(\frac{x-\mu}{\sigma}\right)^{2}} \\
& \approx e^{-20(x-1)^{2}}
\end{aligned}
$$

$\mu$ and $\sigma$ are the mean and standard deviation of the distribution, $\mathrm{x}$ is the continuous value of the shunt as a percentage of it's switched in value. $s$ is a scaling value.

The parameters $s=0.38, \mu=1$ and $\sigma=0.15$ were found to give the required curve shape illustrated in Figure 3.

The following pseudo code describes the probabilistic method, which is executed iteratively as shown by figure 2 . $\mathrm{S}_{\mathrm{ci}}$ represents the continuous solution of the $i$ th shunt during the iteration. $\mathrm{S}_{\mathrm{mi}}$ represents the discrete value of the $i$ th shunt. $S_{p i}$ is a flag determining the optimization status of the $i$ th shunt. $\mathrm{S}_{\mathrm{pi}}=1$ means the ith shunt is an optimisable control and $\mathrm{S}_{\mathrm{pi}}=0$ means the ith shunt is frozen out and no longer optimisable.

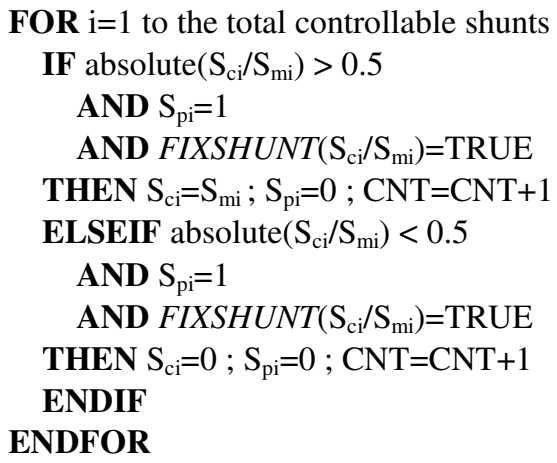

FUNCTION FIXSHUNTS (y)

$\mathrm{R}=$ Random number between 0 and 1

IF absolute $(\mathrm{y})>0.5$

AND $\mathrm{R}<\exp \left(-20(\mathrm{y}-1)^{2}\right) *$ ScaleFact

THEN Return TRUE

ELSEIF absolute $(\mathrm{y})<0.5$

AND $\mathrm{R}<\exp \left(-20 \mathrm{y}^{2}\right) *$ ScaleFact

THEN Return TRUE

ELSE Return FALSE

where CNT is a counter that returns the number of shunts fixed during an iteration of the heuristic. ScaleFact is a scaling factor that multiplies the probability of shunt fixing. A high value of ScaleFact will cause the heuristic to have a small number of iterations compared to a low value that will provide a better solution with more iterations.

The probabilistic method has just two adjustable parameters - probability factor and maximum number of iterations. The probability factor scales the probability of fixing, so a low value means that the technique will take longer to fix all the shunts.

\section{Adaptive Threshold Technique}

The adaptive threshold technique also examines the continuous solution of each optimisable discrete control at every iteration as shown in Figure 2, fixing a subset of the shunts to their nearest discrete value. A shunt is fixed if the continuous solution is within a threshold distance of a

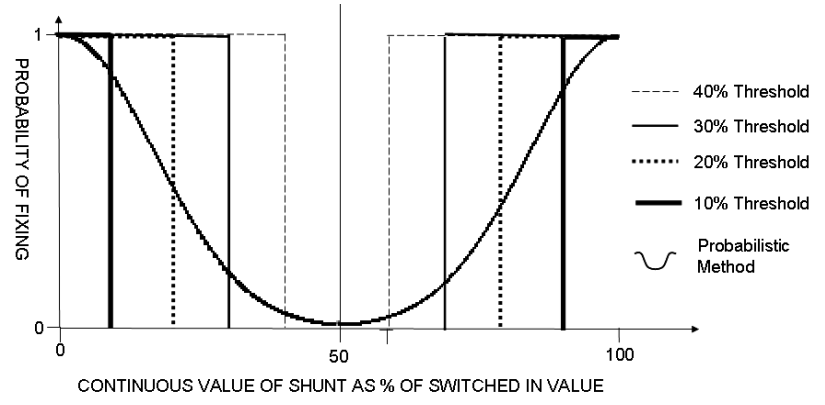


Fig. 3 Probability of fixing in the probabilistic technique and the thresholds used in the threshold technique.

discrete value. The first iteration of the adaptive threshold method fixes only those shunts that are within a $10 \%$ threshold from their discrete value, the second iteration applies a threshold of $20 \%$, the third iteration applies a threshold of $30 \%$ and then the ten remaining iterations apply threshold of $40 \%$.

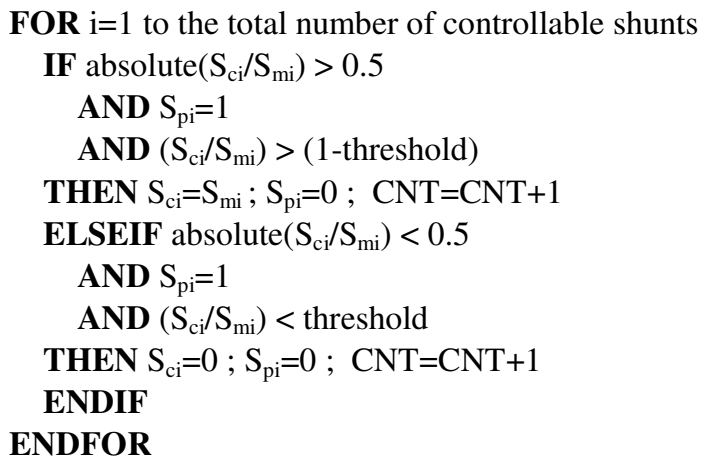

\section{Measuring The Performance Of The Proposed METHODS}

\section{A. Network Models}

Widely recognised standard network test cases ranging in size from 6 to 118 buses were modified to evaluate the performance of the proposed SCORPF extension. The 6 bus network was the Ward Hale 6 bus test case [9], while the other networks were all standard IEEE test cases [10]. Additional switchable capacitors and reactors were modelled at PQ buses with sizes determined by twice the requirement for reactive support at the corresponding bus, thus making the optimization more challenging. Both the 57 bus and 118 bus security constrained network test cases were modelled with 6 and 16 contingencies respectively each contingency contained the outage of a single transmission line. The transmission lines were chosen in the order of those with the largest pre-fault flow. Table I lists the shunts added to each standard network test case. Table II lists parameters describing every network that was tested. It is useful to gauge the change in the losses objective when switching a single shunt. On the IEEE 57 bus network switching the smallest capacitor caused a $0.94 \%$ reduction and switching the largest capacitor caused a $1.4 \%$ increase. Transformer taps were not flagged as optimisable and were treated as continuous controls. This approach is acceptable in practice since the discrete steps of transformers are much smaller than the block size of the switchable shunts [16].

In addition four practical large scale transmission network models were used to evaluate the performance of the proposed SCORPF extensions. Each GB transmission network model was a manually secured day-ahead system representation with topology, demand, generation and controls all representative of what was likely to occur on the real system. The shunts were initialized to a flat start for the purposes of testing the proposed techniques. Typically each of these network models consisted of around 3500 nodes, 3000 lines, 2500 transformers, 80 continuous generator voltage target controls, 20 static VAr compensator (SVC) voltage set-points and 270 discrete shunt controls.

TABLE I

MOdiFicATIONS TO THE STANDARD NETWORK TEST CASES

\begin{tabular}{l|l}
\hline $\begin{array}{c}\text { Name of } \\
\text { System }\end{array}$ & $\begin{array}{l}\text { List of shunt MVAR sizes added to the standard network models } \\
\text { (Bus number to which additional shunt is connected) }\end{array}$ \\
\hline Ward and Hale 6 Bus & $\begin{array}{l}0.4(5), 0.2(5), 0.1(5), 0.05(5), 0.025(5), \\
0.0125(5), 0.00625(5), 0.5(6), 0.25(6), 0.125(6),\end{array}$ \\
& $0.0625(6), 0.03125(6), 0.015625(6), 0.007813(6)$ \\
\hline IEEE 14 & $-40(4), 14.4(10), 4.85(11), 4(12), 13.2(13), 10.7(14)$ \\
\hline IEEE 30 & $15(3), 13(7), 36(12), 3.5(14), 5.5(15), 5(16), 6.4(17), 1.8(18)$ \\
& $6.4(19), 16(21), 4(24), 0.45(25), 4.5(26), 15(27), 2.2(29), 5(30)$ \\
& $8(22), 3.5(23)$ \\
\hline IEEE 57 & $-95(4),-120(10),-72(11),-50(13),-110(14), 25(16), 34(17)$ \\
& $130(18), 35(20),-35(21), 20(38), 80(41), 8(42), 10(44), 90(45)$ \\
& $120(46), 24(47), 62(49), 22(50), 129(51), 18(52), 12(53)$ \\
& $50(55), 17(56), 18(57)$ \\
\hline IEEE 118 & $-87(5), 45(15), 74(3), 30(9), 58(11), 35(13),-225(17), 22(20)$, \\
& $17(21), 15(22), 20(23), 17(28), 300(30), 20(33), 28(35)$, \\
& $187(38), 21(39), 21(41),-13(44), 19(45),-13(48), 24(51)$, \\
& $11(52), 21(53), 45(60), 400(63), 300(64), 26(67), 300(68)$, \\
& $42(71), 86(75), 115(78), 26(79), 160(81), 15(83), 36(84)$, \\
& $34(86), 69(88), 32(93), 38(94), 64(95), 15(97), 10(98), 29(101)$, \\
& $36(102), 53(106), 33(114), 14(115), 15(117), 105(118)$
\end{tabular}

TABLE II

PARAMETERS DESCRIBING THE STANDARD NETWORKS AND FULL GB NETWORK TEST CASES

\begin{tabular}{|l|l|l|l|l|}
\hline $\begin{array}{l}\text { Name of } \\
\text { System }\end{array}$ & $\begin{array}{l}\text { Number of } \\
\text { Buses } \\
\text { /Branches }\end{array}$ & $\begin{array}{l}\text { Number of } \\
\text { Generators }\end{array}$ & $\begin{array}{l}\text { Number of } \\
\text { Discrete Controls } \\
\text { Continuous Controls }\end{array}$ & $\begin{array}{l}\text { Number of modelled } \\
\text { Contingencies }\end{array}$ \\
\hline Ward-Hale 6 & $6 / 8$ & 2 & $14 / 0$ & 0 \\
\hline IEEE 14 & $14 / 20$ & 5 & $6 / 0$ & 0 \\
\hline IEEE 30 & $30 / 41$ & 6 & $18 / 0$ & 0 \\
\hline IEEE 57 & $57 / 80$ & 7 & $25 / 0$ & 6 \\
\hline IEEE 118 & $118 / 186$ & 54 & $50 / 0$ & 0 \\
\hline GB 1 & $3465 / 5213$ & 223 & $263 / 73$ & 0 \\
\hline GB 2 & $3587 / 5397$ & 303 & $300 / 94$ & 0 \\
\hline GB 3 & $3450 / 5186$ & 234 & $262 / 80$ & 40 \\
\hline GB 4 & $3551 / 5393$ & 253 & $269 / 81$ & \\
\hline
\end{tabular}

\section{B. Baseline}

The network models described in table I and table II were used to evaluate the performance of the proposed techniques. The solutions from the proposed techniques were compared against the initial starting point power flow solution, optimization with shunt rounding and against a non-feasible optimization solution in which all controls were assumed to be continuously varying. The solutions from the proposed techniques were also compared against the solution from the mixed integer non-linear programming algorithm MINLP developed by Fletcher and Leyffer [11]. This mathematical programming method implements a branch-and-bound technique that searches a tree with nodes corresponding to continuous non-linearly constrained optimization problems. The MINLP algorithmic method utilizes branch and bound, interlacing the continuous and integer optimization problems so that the non-linear part of the problem is solved while searching the branching tree. In 
order to achieve this the MINLP algorithmic method implements early branching and does not therefore solve the non-linear problem to optimality at each branch point, which has the possible drawback that the overall optimal solution may not be found.

All standard network test cases up to 118 buses were converted into AMPL code, which is a modelling language for linear and non-linear optimization problems, this code can be interpreted by the MINLP solver. Network constraints and controls were implemented to identically represent the constraint and controls solved by the SCORPF extension techniques.

The practical large scale GB power network test cases could not be encoded in AMPL, so MINLP results could not be derived. The proposed SCORPF extensions were therefore compared to the 2 staged SCORPF - continuous SCORPF is first solved for all variables, which then rounds all discrete controls to their nearest value; this was then followed by another round of optimization utilizing only continuous control variables to remove any violations. These results are labelled 'Rounding' in table III.

\section{Executing the SCORPF extension techniques}

The proposed SCORPF extensions were written in MATLAB version 7.0 on a $\mathrm{P} 43 \mathrm{GHz}$ with $512 \mathrm{MB}$ memory. On a full scale constrained GB transmission network model the SCORPF extension techniques typically took 9 minutes to solve find a solution.

To meaningfully compare the results with the baselines we repeatedly executed the probabilistic technique one hundred times with different random number generator seed states. This allowed the mean value of transmission losses and standard deviation of the solutions to be determined. A maximum of 20 iterations were specified for all the extension techniques. ScaleFact was kept constant at a value of 0.9 throughout the results presented in Table III.

\section{RESUlTS AND ANALYSIS}

Figure 4 shows the losses objective function contour surface of the Ward Hale 6 bus network. The objective function was mapped over a range of susceptance values for shunts attached to bus 5 and bus 6 . The solutions from the standard rounding technique and the adaptive threshold technique were identical as all shunts were switched out. The figure indicates that MINLP has correctly located the minima at $(0.24,0.18)$. The probability factor of the probabilistic technique was varied with the values $0.1,0.5$ and 0.9 - the solutions are marked on the figure as P0.1, P0.5 and P0.9 respectively. The P0.9 and P0.5 probabilistic solutions lie on the flat area close to the optimal MINLP solution.

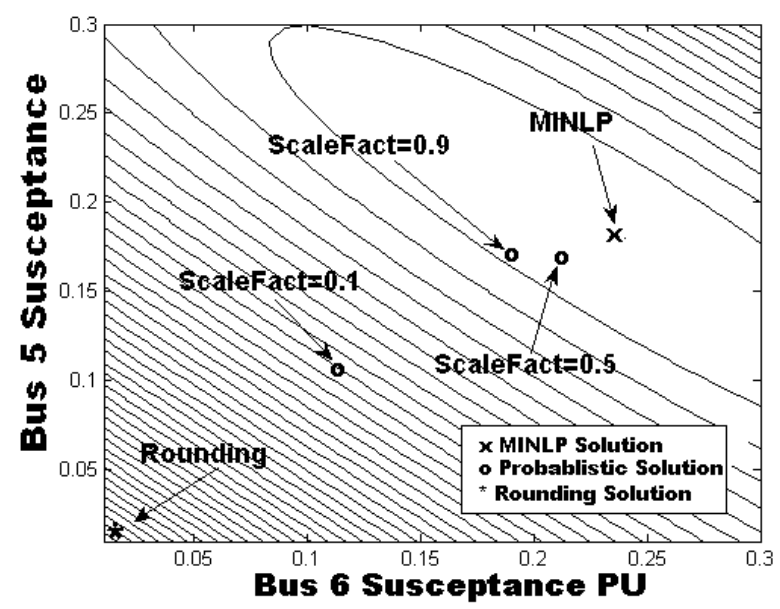

Fig. 4 Losses objective function contour surface - solutions from the probabilistic heuristic are plotted for various values of ScaleFact. These solutions are also compared against the rounding and MINLP solutions.

Table III shows the optimization results of the standard network test cases and four practical large-scale power networks. The first five sets of results are intact network optimizations on standard test networks. The next two sets of results are for the IEEE 57 and IEEE 118 bus networks with contingencies constraining the optimization. The following three sets of results are practical large-scale intact GB network optimisations. The final set of results is a contingency constrained optimization on a GB network. In the large-scale GB network optimizations the full mixed integer optimization problem was tackled, which meant continuous generator voltage target, continuous SVC voltage set-point, discrete shunt capacitor and discrete reactor controls were flagged as optimisable.

Table III allows us to compare the losses solution from the initial power flow, non-feasible SCORPF assuming all controls are continuously varying, the 2 staged SCORPF rounding method, the MINLP solution, the adaptive threshold technique and the probabilistic technique. The last three columns present the percentage improvement scores of the SCORPF extension techniques and MINLP relative to the continuous case. A score of $100 \%$ indicates that the discretization algorithm has performed as well as the nonfeasible continuous solution and $0 \%$ indicates the solution is no better than the rounding method.

The last three columns of Table III can be determined by using the relationship as given below in equation (8) and converting the resulting value to a percentage. 
TABLE III

COMPARISON OF OPTIMIZATION RESULTS FOR THE TEST NETWORKS

\begin{tabular}{|c|c|c|c|c|c|c|c|c|c|}
\hline \multicolumn{7}{|c|}{ P I o s s ( MW ) } & \multicolumn{3}{|c|}{$\begin{array}{l}\text { Reduction } \% \text { in objective } \\
\text { relative to continous case }\end{array}$} \\
\hline & Initial & Continuous & Rounding & MINLP & Adaptive & Averaged Probablistic & \begin{tabular}{|l|} 
Adaptive \\
\end{tabular} & \begin{tabular}{|l|} 
Probabilistic \\
\end{tabular} & MINLP \\
\hline WardHale 6 & 10.210 & 8.674 & 10.213 & 8.747 & 10.213 & $8.912+/-0.16$ & 0.0 & 84.5 & 95.3 \\
\hline IEEE 14 & 13.358 & 13.240 & 13.358 & 13.334 & 13.358 & $13.334+/-0.14$ & 0.0 & 20.3 & 20.3 \\
\hline IEEE 30 & 17.542 & 17.213 & 17.542 & 17.303 & 17.542 & $17.316+/-0.03$ & 0.0 & 68.7 & 72.6 \\
\hline IEEE 57 & 28.620 & 25.720 & 28.620 & 27.924 & 28.620 & $27.386+/-0.26$ & 0.0 & 41.6 & 26.9 \\
\hline IEEE 118 & 132.483 & 128.380 & 132.480 & \#\# & 132.140 & $131.296+/-0.41$ & 8.3 & 28.9 & \#\# \\
\hline IEEE 57 + 6C & 28.620 & 25.760 & 27.860 & $\#$ & 27.860 & $27.281+/-0.29$ & 0.0 & 27.6 & $\#$ \\
\hline IEEE 118 + 16C & 132.483 & 128.390 & 132.610 & $\#$ & 132.110 & $131.160+/-0.45$ & 11.8 & 34.4 & $\#$ \\
\hline GB1 & 881.90 & 830.21 & 839.69 & $\#$ & 834.93 & $830.00+/-0.47$ & 50.2 & 102.2 & $\#$ \\
\hline GB2 & 1414.20 & 1322.07 & 1368.39 & $\#$ & 1322.86 & $1323.38+/-0.52$ & 98.3 & 97.2 & $\#$ \\
\hline GB3 & 916.69 & 890.83 & 902.54 & $\#$ & 894.69 & $892.57+/-0.47$ & 67.0 & 85.2 & $\#$ \\
\hline GB4 + 40C & 1239.68 & 1136.31 & 1139.46 & $\#$ & 1139.50 & $1136.83+/-2.54$ & -1.3 & 83.5 & $\#$ \\
\hline
\end{tabular}

$\mathrm{C}=$ contingencies, \#=MINLP result not calculated, \#\#=MINLP timed out, *=see text for calculation explanation.

Rounded_Solution-Solution

Rounded_Solution-Continuous_solution

Where solution is the magnitude of power losses from the adaptive threshold, probabilistic or MINLP technique. continuous_solution is the magnitude of power losses in the non-feasible SCORPF continuous solution and rounded_solution is the magnitude of power losses in the 2 staged SCORPF rounding method. For example consider the reduction in the objective function for the Probabilistic case relative to the Continuous case as presented as presented in Table III for the WardHale 6 bus test network. This can be determined as a fraction or percentage as follows:

$\left(\frac{10.213-8.912}{10.213-8.674}\right)=1.301 / 1.539=0.845$ or $84.5 \%$.

The above percentage value is presented in the penultimate column of Table III for the WardHale 6 bus test network.

On the standard network test cases the rounding method did not manage to achieve a reduction in real power losses because the continuous solution of the shunt devices was just below $50 \%$ and therefore rounding switched out these devices. In general the adaptive threshold technique did not manage to reduce real power losses on the standard network test cases because the solution value of the shunt devices was always outside of a threshold band. However, the probabilistic technique managed to reduce the real power losses by an average of $3.6 \%$. Since the probabilistic technique is based on random numbers it essential to run multiple executions to assess its performance. Table IV presents a statistical analysis - notice that the standard deviation is small and the worst case values are still better than the traditional 2 staged SCORPF rounding method.
TABLE IV

STATISTICS OF VARIATION IN PROBABLISTIC TECHNIQUE'S SOLUTION

\begin{tabular}{|l|c|c|c|}
\hline $\mathbf{P}_{\text {loss }}(\mathbf{M W})$ & $\mathbf{6}$ bus & IEEE 57 bus & IEEE 118 bus \\
\hline Average & 8.912 & 27.386 & 131.296 \\
\hline Standard Deviation & 0.163 & 0.261 & 0.410 \\
\hline Best & 8.747 & 27.109 & 130.790 \\
\hline Worst & 9.862 & 28.409 & 132.270 \\
\hline
\end{tabular}

Figure 5 indicates how the losses evolved with iteration number on the 6 bus, 57 bus and 118 bus networks. A similar evolution of the losses value can be seen on both networks. At each iteration of the SCORPF extension a number of shunts are fixed to their nearest discrete value, which typically causes a slight increase in losses. Analysis of the shunt switching pattern revealed that the large rise in losses near the end of the iterative process was due to shunts in the middle of their range being fixed. Figure 6 illustrates the effect on the averaged probabilistic solution, for the 57 bus and 118 bus networks, when the probability factor was varied. The error bars indicate the standard deviation in the solution value. It can be seen that the 6 bus network results varied smoothly with a minimum average losses and low standard deviation at a probability factor of 0.9 . The results on the 118 bus network were less smooth, but still indicated that a probability factor of 0.9 was reasonable. For comparison the results from the 2 staged SCORPF rounding method are also included in figure 6 indicated by the horizontal lines. 


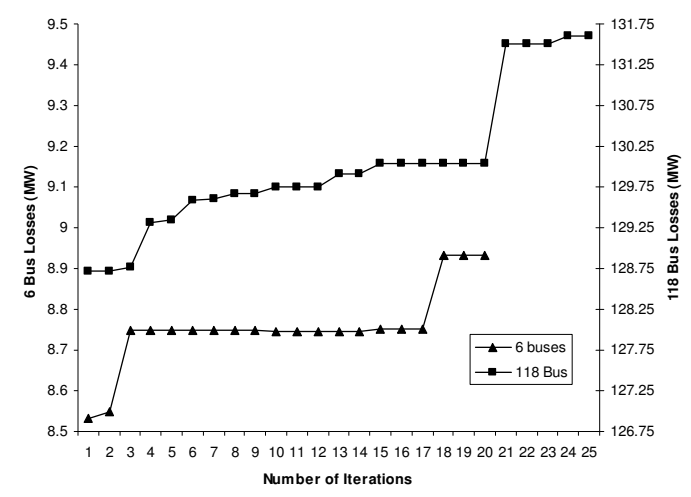

Fig. 5 Variation of real power losses with the number of iterations of the probabilistic technique.

MINLP was used to solve the standard network test case discrete optimization problems; MINLP does not scale well and cannot therefore efficiently calculate the solution to larger problems or problems involving a large number of contingencies. Where a MINLP result has been determined, the probabilistic technique achieved at least $98 \%$ of the losses reduction achieved by MINLP.

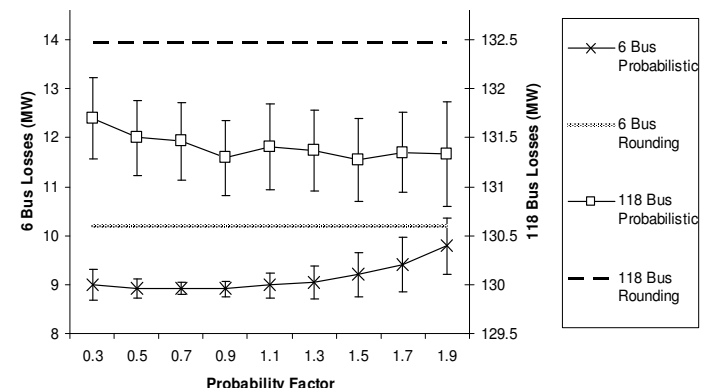

Fig. 6 Illustrates the effect on the averaged probabilistic solution, for the 6 bus and 118 bus networks, when the probability factor was varied. The error bars indicate one standard deviation.

The probabilistic technique found a solution better than MINLP on the 57 bus network case. The proposed extension techniques were then tested on more difficult problems involving larger networks and networks involving a number of contingencies. The results show that the proposed techniques generally achieved a significant improvement in comparison to the 2 staged SCORPF.

The CPU time for MINLP to find a solution was recorded and compared to the enhanced SCORPF method time. The MINLP algorithm, which is based on branch and bound, is time exponential in the number of variables. This relationship is confirmed in Figure 7. The performance of the probabilistic technique was worse than MINLP on the Ward Hale 6 and IEEE 14 bus network test cases; however the performance on the IEEE 30 and 57 bus network test cases was significantly better than MINLP. The ratio of the time taken to solve the IEEE 57 bus network problem to the time taken to solve the Ward Hale network problem was 2.2 and 6,200 for the probabilistic technique and MINLP respectively.

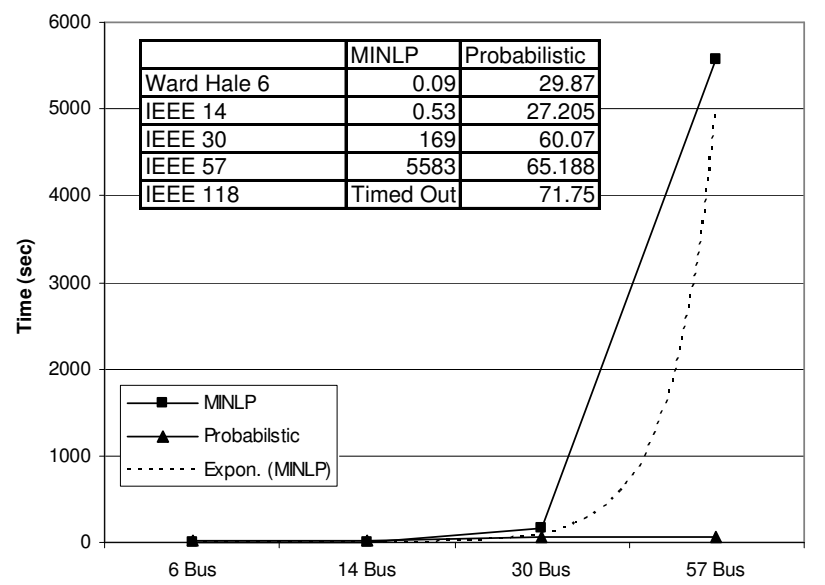

Fig. 7. CPU time to solve the standard network integer optimization problems using MINLP and the probabilistic technique. An exponential best fit line of the MINLP results is shown.

TABLE V

SCALING COMPARISON WITH PENALTY BASED TECHNIQUE Proposed probablistic technique

\begin{tabular}{|l|r|r|r|r|r|}
\hline Discrete variables & 6 & 14 & 21 & 50 & 262 \\
\hline Time(sec) & 30 & 27 & 60 & 72 & 377 \\
\hline Ratio time/variables & 5.00 & 1.93 & 2.86 & 1.44 & 1.44 \\
\hline
\end{tabular}

Penalty based technique - ref [5]

Penalty based technique - ref [5]
\begin{tabular}{|l|r|r|r|r|r|}
\hline Discrete variables & 6 & 13 & 14 & 18 & 162 \\
\hline Time $(\mathrm{sec})$ & 2 & 7 & 4 & 25 & 965 \\
\hline Ratio time/variables & 0.33 & 0.54 & 0.29 & 1.39 & 5.96 \\
\hline
\end{tabular}

Table $\mathrm{V}$ presents a comparison of the scalability of the proposed probabilistic technique with the penalty function based technique of Liu et al. [5], which was described in section II. The authors also customised a selection of standard test networks with discrete controls. The customizations are different because the authors were also investigating discrete transformer taps while this paper has concentrated on solving the large scale discrete shunt despatch problem. Although it is not possible to directly compare results it is possible to compare the scalability of the algorithms as the number of discrete variables increased. The ratio of time / number of discrete variables indicates the scalability. In the case of the probabilistic technique this ratio reduces as the problem size gets bigger indicating good scalability in comparison to the penalty based technique where this ratio gets larger indicating poorer scalability.

Using the adaptive threshold technique on practical largescale GB networks an average improvement score of 54\% was achieved, the probabilistic technique achieved an average improvement score of $92 \%$. The improvement in the losses objective function was $1.15 \%$ and $1.40 \%$ for the adaptive threshold and probabilistic techniques, respectively. On the GB4 network with 40 contingencies the adaptive threshold technique was found to cause a slight increase in the losses, which could be due to a combination of the ineffectiveness of the adaptive threshold technique to find a good solution on this problem and the non-convex nature of the optimization problem. 


\section{CONCLUSIONS}

The SCORPF extension techniques presented in this paper address the practical problem of determining the optimal switching of large numbers of discrete devices. The proposed techniques have been demonstrated to work effectively with an existing SCORPF application, and should therefore be quite easy to integrate directly within these applications. Both of the proposed enhanced SCORPF techniques fixed a subset of the floating optimisable shunts to their nearest discrete at each iteration. The probabilistic technique fixed a shunt based on a probability determined by the closeness of its continuous solution to a discrete value and the adaptive threshold technique fixed a shunt if its continuous solution was inside a threshold.

The probabilistic technique consistently performed well on both the standard network test cases and the practical largescale GB networks, the adaptive threshold technique produced an improvement on large-scale GB network cases. Results on standard network test cases up to 118 buses have shown that the probabilistic technique can achieve results that are sometimes better than MINLP, and a significant improvement on rounding the shunts to their nearest discrete value at the final iteration of the optimization. More research is required to fully quantify the practical benefit of the proposed techniques on a range of practical large-scale GB networks that include large numbers of contingencies. Investigation of the scope for enhancing the performance of the proposed techniques will be required. Further work could also include the development of a hybrid heuristic approach.

\section{ACKNOWLEDGMENT}

The authors acknowledge the support from Nexant for reviewing this paper. The authors also acknowledge the use of and user support for Nexant's SCOPE software, which was used to perform the SCORPF analysis in this research. Finally the authors wish to acknowledge the financial assistance and support of National Grid and the EPSRC.

\section{REFERENCES}

[1] P. Macfie, G.A. Taylor, M.R. Irving, P. Hurlock, M.E. Bradley, R. Morfill and H.-B. Wan, "Operational Analysis of Security Constrained Optimal Reactive Power Flow Solutions", in Proc IEEE PES General Meeting, Calgary, Canada, PESGM2009-001137, 26-30 July, 2009.

[2] K. Karoui, L. Platbrood, H. Crisciu1, and R. Waltz, "New Large-scale Security Constrained Optimal Power Flow program using a new Interior Point algorithm", in Proc European Electricity Market International Conf., pp1-6, Lisbon, Portugal, May 2008.

[3] J. Ramos, A. Exposito, and V. Quintana, "Transmission power loss reduction by interior-point methods: implementation issues and practical experience", in Proc IEEE Generation, Transmission and Distribution Conf., Vol. 152, pp90-98, January 2005.
[4] R. Jabr, "Optimal placement of capacitors in a radial network using conic and mixed integer linear programming", Electric Power System Research, Vol. 78, pp941-948, 2008.

[5] M. Liu, S. Tso, and Y. Cheng, “An Extended Nonlinear Primal-Dual Interior-Point Algorithm for Reactive-Power Optimization of LargeScale Power Systems With Discrete Control Variables", IEEE Transactions on Power Systems, Vol. 17, pp982-994, November 2002 .

[6] I. Silva, and S. Carneiro, "A Heuristic Constructive Algorithm for Capacitor Placement on Distribution Systems", IEEE Transactions on Power Systems, Vol. 23, pp1619-1626, November 2008.

[7] W. Zhang, and L. Tolbert, "Survey of Reactive Power Planning Methods", in Proc IEEE PES General Meeting, Vol. 2, pp1430-1440, San Francisco, US 12-16th June 2005.

[8] S. Lin, Y. Ho, and C. Lin, "An Ordinal Optimization Theory-Based Algorithm for Solving the Optimal Power Flow Problem With Discrete Control Variables", IEEE Transactions on Power System, Vol. 19, no. 1, pp276-286, Feb. 2004.

[9] Z. H. Bie, Y. H. Song, and X. F. Wang, G. A. Taylor, M. R. Irving, “A Transition-Optimized Approach to Reactive Power and Voltage Control", in Proc IEEE PES General Meeting, Vol. 1, pp 226-232, Denver, Colorado, US, 6-10th June 2004.

[10] IEEE standard test networks [online]. Available: http://www.ee.washington.edu/research/pstca/

[11] R. Fletcher and S. Leyffer, "Solving mixed integer nonlinear programs by outer approximation”, Mathematical Programming, Vol. 66, pp 327-349, Dec. 1994.

[12] Z. H. Bie, Y. H. Song, and X. F. Wang, G. A. Taylor, M. R. Irving, "Integration of Algorithmic and Heuristic Techniques for TransitionOptimised Voltage and Reactive Power Control", in IEE Proceedings - Generation, Transmission and Distribution, Issue 2, Vol. 153, pp 205-210, 2006.

[13] G.A. Taylor, S. Phichaisawat, M.R. Irving and Y.-H. Song, "Voltage Security and Reactive Power Management", IMA Journal of Management Mathematics., Vol. 15, pp 369-386, 2004.

[14] Y. Zhang, Z. Ren, "Real-time optimal reactive power dispatch using multi-agent technique", Electric Power System Research, Vol 69, pp 259-265, 2004.

[15] J. Chiou, C. Chang, C. Su, "Ant Direction Hybrid Differential Evolution for Solving Large Capacitor Placement Problems", IEEE Transactions on Power Systems, Vol. 19, pp 1794-1800, 2002.

[16] A. D. Papalexopoulos, C. F. Imparato, and F. F. Wu, "Large Scale Optimal Power Flow : Effects of Initialization, Decoupling, and Discretization”, IEEE Trans. on PWRS, pp 748-759, 1989.

[17] H.-D. Chiang, J.-C. Wang, O. Cockings, "Optimal capacitor placements in distribution systems - Part 2: solution algorithms and numerical results", IEEE Trans. Power Deliv. 5, pp 643-649, 1990.

[18] S. Sundhararajan, A. Pahwa, "Optimal selection of capacitors for radial distribution systems using a genetic algorithm", IEEE Trans. Power Syst. 9, pp 1499-1507, 1994.

[19] A. Monticelli, "State estimation in electric power systems: a generalized approach", Springer, pp 76, 1999. 


\section{BIOGRAPHIES}

Peter Macfie received his MPhys degree from the University of Southampton in 2004 and MSc degree from the University of Surrey in 2005. In 2006 he started his Engineering Doctorate research degree with Brunel University, Uxbridge, UK. He is currently based full-time at the National Grid Control Centre, Wokingham, UK.

Paul Hurlock received his BSc degree from the University of Portsmouth in 1999 and joined National Grid the same year. Since then he has progressed through operational and commercial post-event review to his current position as a team leader within the BSIS Strategy team at the National Grid Control Centre, Wokingham, UK.

Gareth Taylor received his BSc degree from the University of London, UK in 1987 and MSc and PhD from the University of Greenwich, UK in 1992 and 1997, respectively. He was the National Grid UK post-doctoral scholar at Brunel University, UK from 2000-2003. He is currently a senior lecturer and course director within the Brunel Institute of Power Systems, Brunel University, UK. His research interests include power system and network optimization.

Hai-Bin Wan received his BEng and MPhil from Northeastern University, China in 1986 and 1989 respectively, and PhD from University of Bath, UK in 1997. His research interests include power system management and control, electricity market and system optimization.

Malcolm Irving is Professor of Power Systems in the School of Engineering and Design at Brunel University, Uxbridge, UK. He is also Director of the Brunel Institute of Power Systems, a major research group in the University specialising in algorithms and software for generation, transmission and distribution systems. He is the author or co-author of more than 100 research publications in the fields of power network analysis and optimal power system operation. 